\title{
WARM DUST IN THE TERRESTRIAL PLANET ZONE OF A SUN-LIKE PLEIADES STAR: COLLISIONS BETWEEN PLANETARY EMBRYOS?
}

\author{
Joseph H. Rhee, ${ }^{1}$ Inseok Song, ${ }^{2}$ and B. Zuckerman ${ }^{1,3}$ \\ Received 2007 August 10; accepted 2007 October 18
}

\begin{abstract}
Only a few solar-type main-sequence stars are known to be orbited by warm dust particles; the most extreme is the G0 field star BD +20307 that emits 4\% of its energy at mid-infrared wavelengths. We report the identification of a similarly dusty star HD 23514, an F6-type member of the Pleiades. A strong mid-IR silicate emission feature indicates the presence of small warm dust particles, but with the primary flux density peak at the nonstandard wavelength of $\sim 9 \mu \mathrm{m}$. The existence of so much dust within an AU or so of these stars is not easily accounted for given the very brief lifetime in orbit of small particles. The apparent absence of very hot $(\gtrsim 1000 \mathrm{~K})$ dust at both stars suggests the possible presence of a planet closer to the stars than the dust. The observed frequency of the BD +20 307/ HD 23514 phenomenon indicates that the mass equivalent of Earth's Moon must be converted, via collisions of massive bodies, to tiny dust particles that find their way to the terrestrial planet zone during the first few hundred million years of the life of many (most?) Sun-like stars. Identification of these two dusty systems among youthful nearby solar-type stars suggests that terrestrial planet formation is common.
\end{abstract}

Subject headings: circumstellar matter — infrared: stars — open clusters and associations: individual (Pleiades) — planetary systems: formation - stars: individual (HD 23514)

Online material: color figure

\section{INTRODUCTION}

The Spitzer Space Telescope is now providing a wealth of new information about dusty stars in the Milky Way. However, because Spitzer is a pointed telescope, during its cryogenic lifetime it will examine only a modest portion of the sky, about $1 \%$. For the very rare, very infrared-bright, nearby field star, the less sensitive Infrared Astronomical Satellite (IRAS) was actually the better search engine because it was an all-sky survey. Consequently, we have an ongoing program to correlate the IRAS catalog with the Hipparcos (Song et al. 2002; Rhee et al. 2007b) and Tycho catalogs (Melis et al. 2008). To date we have identified at least three nearby stars of age $\gtrsim 100 \mathrm{Myr}$ that emit at least a few percent of their energy at infrared wavelengths. These are field star BD +20 307 (Song et al. 2005; A. Weinberger et al. 2008, in preparation), a member of a field Tycho binary star (Melis et al. 2008), and the Pleiades star HD 23514 (=HII 1132) that is the principal focus of the present paper. In addition to these three stars, BP Psc, discovered by IRAS to be very bright at far-IR wavelengths, might also be not young and fairly nearby (Zuckerman et al. 2007). In contrast, to the best of our knowledge, Spitzer has not yet discovered any nearby star not in a region of recent star formation nearly as infrared luminous as these four. By infrared luminosity we mean the fraction of a star's bolometric luminosity as seen from Earth that is absorbed and reradiated by dust particles. For the above-mentioned four stars this fraction is in the range between $2 \%$ and $75 \%$.

When the remarkable properties of BD +20 307 were first appreciated, a statistical analysis of the frequency of occurrence

\footnotetext{
${ }^{1}$ Department of Physics and Astronomy, University of California, Los Angeles, CA 90095-1562; rhee@astro.ucla.edu, ben@astro.ucla.edu.

2 Spitzer Science Center, IPAC, California Institute of Technology, Pasadena, CA 91125; song@ipac.caltech.edu.

3 NASA Astrobiology Institute.
}

of such very dusty stars, based on only one example, might be regarded as premature (and no such analysis was attempted by Song et al. 2005). However, with the recognition (below) that properties of HD 23514 are quite similar to those of $\mathrm{BD}+20307$, the phenomenon has been transformed from a miracle into a statistic. Consequently, following description of our observations, in $\S 3$ we discuss the occurrence frequency of the very dusty phenomenon and what it might imply for the evolution of planetary systems in orbit around adolescent-age main-sequence stars.

\section{OBSERVATIONS AND RESULTS}

The large mid-infrared excess of HD 23514 (Fig. 1) was discovered by IRAS only in its $12 \mu \mathrm{m}$ band. Due to its large beam size, IRAS measurements often included many contaminating background sources, and some IRAS-identified IR excess stars were subsequently found to be false positives (Song et al. 2002; Rhee et al. 2007b). In addition, all previously known dusty mainsequence stars with excess emission detected at IRAS $12 \mu \mathrm{m}$ also had excess emission in at least one of the longer IRAS 25,60, or $100 \mu \mathrm{m}$ bands. This is because the IR detection of cold Kuiper Belt analogs has been much more frequent than detection of warm asteroid belt analogs. Thus, significant ambiguity among IRAS excess candidates and lack of known main-sequence stars with strong mid-IR excess emission perhaps helped to prolong the overlooking of the IRAS $12 \mu \mathrm{m}$ detection of HD 23514.

Spangler et al. (2001) reported a marginal detection of dust excess emission from HD 23514 at 60 and $90 \mu \mathrm{m}$ with a pointed observation of the Infrared Space Observatory (ISO). They did mention the IRAS $12 \mu \mathrm{m}$ measurement, but then used only ISO data, leading them to an incorrect conclusion about the dust properties of this $\operatorname{star}\left(T_{\text {dust }} \sim 70 \mathrm{~K}, L_{\text {dust }} / L_{*} \sim 3 \times 10^{-4}\right)$. HD 23514 was rediscovered as a potential hot dust star from our search of main-sequence mid-IR excess stars using public Spitzer data (J. H. Rhee et al. 2008, in preparation). Spitzer MIPS $24 \mu \mathrm{m}$ images have a fairly large field of view $\left(5^{\prime} \times 5^{\prime}\right)$, and many field 


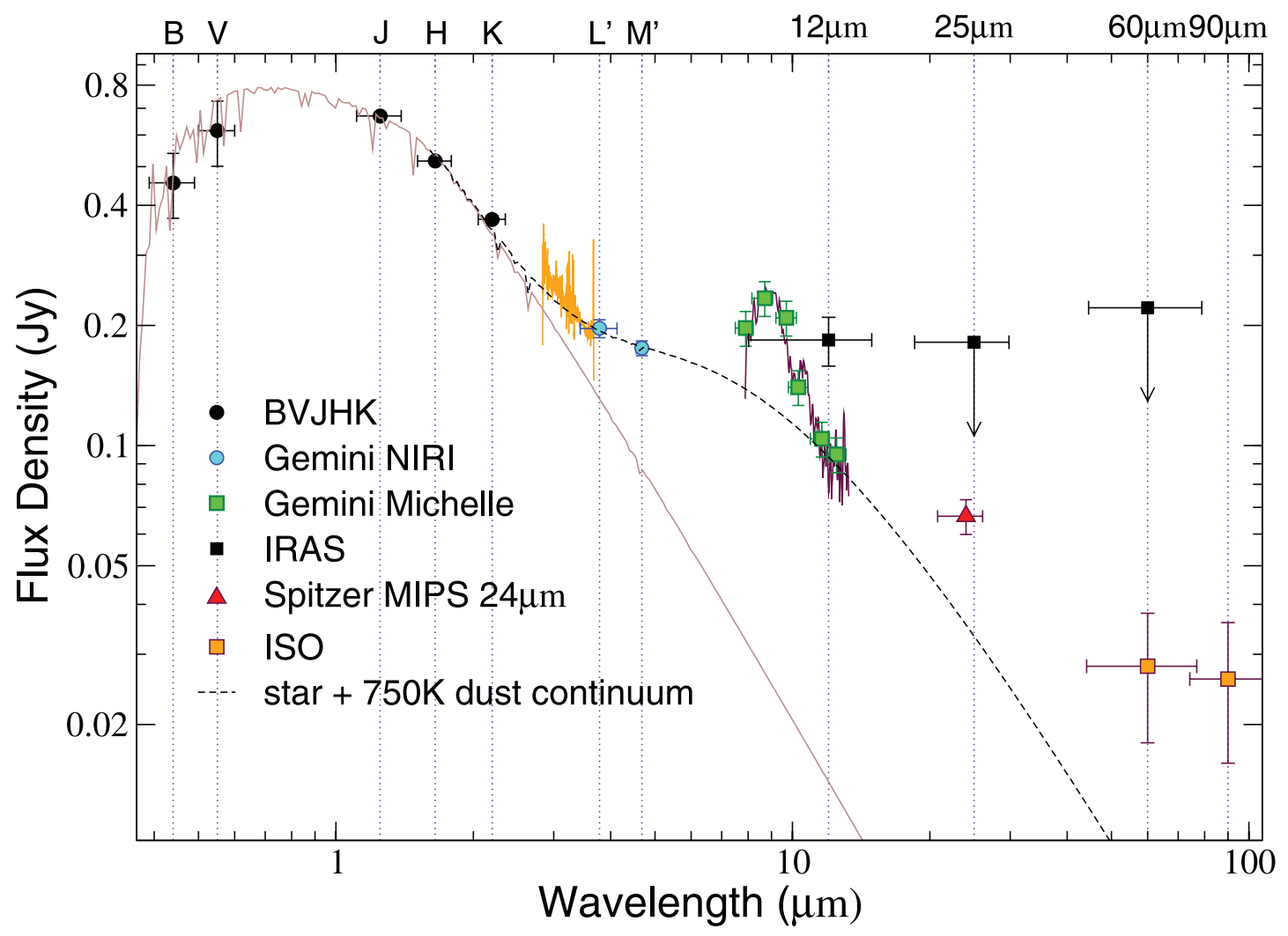

FIG. 1. - SED of HD 23514. The NIRSPEC spectrum is shown in yellow (wavelengths 2.8-3.7 $\mu \mathrm{m}$ ), while the Michelle spectrum is in maroon (7.8-13.3 $\mu \mathrm{m})$. The derived stellar parameters for HD 23514 are given in a note to Table 1. For each measurement the horizontal bars indicate the passband of the filter used and the vertical bars depict $1 \sigma$ flux uncertainties.

stars appear in most MIPS images. HD 23514 was included, serendipitously, in the FEPS (Formation and Evolution of Planetary Systems; Meyer et al. 2004) field of Pleiades star HII 1182.

Follow-up imaging observation of HD 23514 was carried out with the Near-Infrared Imager (NIRI) and Mid-IR Imager/ Spectrometer (Michelle) at Gemini North Telescope. $L^{\prime}(3.8 \mu \mathrm{m})$ and $M^{\prime}(4.7 \mu \mathrm{m})$ images were obtained with NIRI using a fourpoint dithered pattern. The standard "beam switching" mode was used for six mid-IR narrowband images with Michelle by chopping the secondary at $2.7 \mathrm{~Hz}$ and nodding the telescope every $\sim 30 \mathrm{~s}$. For $L^{\prime}$ and $M^{\prime}$ images, dark frames were first subtracted from raw frames. After sky subtraction, images were then flat-fielded using a sky frame made by median combination of dithered images. Images at each band were shifted, added, and averaged to produce a final image at each wavelength. For six mid-IR images, raw images were sky-subtracted using the sky frame from each chop pair. Subtraction of a nodded pair removed the thermal emission from the telescope. Standard stars, HD 22686 and HD 18884, were observed close in time and position to our target and used for absolute flux calibration of $L^{\prime}$ and $M^{\prime}$ images and of six mid-IR images, respectively. Finally, aperture photometry was performed on both HD 23514 and the standard stars to compute flux density at each band. We used aperture radii of $0.96^{\prime \prime}$ and sky annuli of $1.42^{\prime \prime}$ and $1.97^{\prime \prime}$ for both the target and the standard star.

These ground-based images at 3.8-11.7 $\mu \mathrm{m}$ bands show only one object at the expected target location (Gemini blind pointing accuracy is good to $<1^{\prime \prime}$ ), thus verifying that the dust emission shown in Figures 1 and 2 originates from HD 23514. Flux measurements at those wavelengths confirmed excess emission above the stellar photosphere.
Near-IR and mid-IR spectra of HD 23514 were obtained using the Near-Infrared Cross-dispersed Echelle Grating Spectrometer (NIRSPEC; McLean et al. 1998) at Keck II Telescope and Michelle at Gemini North Telescope. NIRSPEC was used in a low-resolution mode $(R \sim 2000)$ with the $42^{\prime \prime} \times 0.570^{\prime \prime}$ slit to obtain a $K L(2.8-3.7 \mu \mathrm{m})$ band spectrum of HD 23514. For $N$-band spectra, the low-resolution spectroscopic mode $(R \sim 200)$ of Michelle was used with a 2 pixel wide $\left(0.402^{\prime \prime}\right)$ slit. The $N$-band filter with a central wavelength of $10.5 \mu \mathrm{m}$ was selected to give wavelength coverage of 7.7-14 $\mu \mathrm{m}$. REDSPEC, an IDL-based reduction package for NIRSPEC, was used for the reduction of the NIRSPEC $K L$ spectrum. Dark frames were first removed from raw frames. Using the sky frame of the nod pair for the $K L$ spectrum and of the chop pair for the $N$-band spectrum, the resultant frames were sky-subtracted and flat-fielded. After the spectra of the standard stars (HD 210501 for $K L$-band spectra and HD 18884 for $N$-band spectra) were divided by Planck curves with each star's effective temperature (6400 K for HD 210501 and $3400 \mathrm{~K}$ for HD 18884), these ratioed spectra were then divided into the spectra of HD 23514 to remove telluric and instrumental signatures. Wavelength calibration was performed using an argon lamp spectrum for the NIRSPEC $K L$ spectrum and using atmospheric transition lines from an unchopped raw frame for the Michelle $N$-band spectrum. Finally, photometry values at NIRI $L^{\prime}$ band and Michelle $8.8 \mu \mathrm{m}$ band were used to flux-calibrate $K L$ - and $N$-band spectra, respectively. Near-IR and mid-IR photometry of HD 23514 from both ground- and space-based observations is listed in Table 1.

We note that $M S X$ detected HD 23514 in its A band $(8.28 \mu \mathrm{m}$ isophotal wavelength) with a catalog flux of $158 \mathrm{mJy}$ (color uncorrected) significantly below our measured fluxes from the 


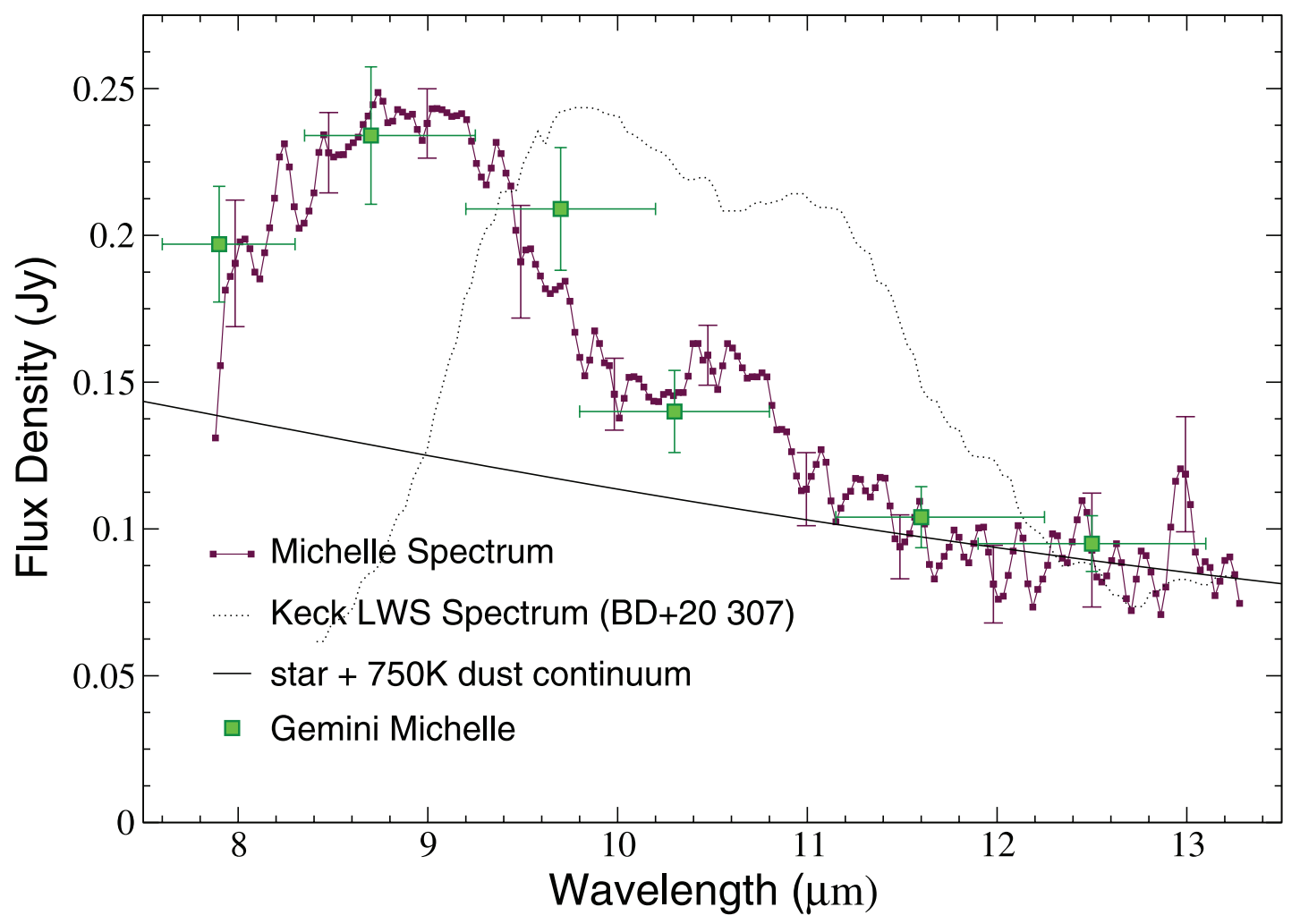

FIG. 2.-Michelle mid-IR spectrum of HD 23514. A 2" wide sky region $\sim 3$ " from the HD 23514 spectrum was used to compute the uncertainty at each wavelength. Representative $1 \sigma$ uncertainties of the spectrum (vertical bars) are given at each half-micron. For comparison, the mid-IR (dotted line) spectrum of BD +20307 is reproduced from Fig. 1 in Song et al. (2005). The spectrum of HD 23514 has almost twice the spectral resolution as the Keck LWS spectrum of BD +20 307. For each photometry measurement ( green square) the horizontal bars indicate the passband of the filter used and the vertical bars depict $1 \sigma$ uncertainties. As noted in $\S 2$, the shape and location of the $\mathrm{BD}+20307$ feature are similar to those usually seen in pre-main-sequence stars and solar system comets.

TABLE 1

HD 23514 NeAr-IR AND Mid-IR Pнotometry

\begin{tabular}{|c|c|c|c|c|}
\hline Filter & $\begin{array}{c}\text { Central Wavelength } \\
(\mu \mathrm{m})\end{array}$ & $\begin{array}{l}\text { Flux Density } \\
\text { (mJy) }\end{array}$ & $\begin{array}{l}\text { Uncertainty } \\
\text { (mJy) }\end{array}$ & Instrument \\
\hline B .................. & 0.44 & $466^{\mathrm{a}}$ & $110^{\mathrm{b}}$ & TYCHO-2 \\
\hline$V \ldots \ldots \ldots \ldots$ & 0.55 & $600^{\mathrm{a}}$ & $86^{\mathrm{b}}$ & TYCHO-2 \\
\hline J .................. & 1.25 & 670 & 15 & 2MASS \\
\hline$H \ldots \ldots \ldots \ldots . . .$. & 1.65 & 516 & 11 & 2MASS \\
\hline$K_{s} \ldots \ldots \ldots \ldots$ & 2.20 & 369 & 9 & 2MASS \\
\hline$L^{\prime} \ldots \ldots \ldots \ldots$ & 3.78 & 196 & 10 & Gemini NIRI \\
\hline$M^{\prime} \ldots \ldots \ldots \ldots$ & 4.68 & 175 & 7 & Gemini NIRI \\
\hline $\mathrm{Si}-1 \ldots \ldots \ldots$ & 7.7 & 197 & 10 & Gemini Michelle \\
\hline Si-2 .......... & 8.8 & 234 & 12 & Gemini Michelle \\
\hline Si-3 .......... & 9.7 & 209 & 10 & Gemini Michelle \\
\hline $\mathrm{Si}-4 \ldots \ldots \ldots$ & 10.3 & 140 & 7 & Gemini Michelle \\
\hline Si-5 .......... & 11.6 & 104 & 5 & Gemini Michelle \\
\hline Si- $6 \ldots \ldots \ldots . .$. & 12.5 & 95 & 5 & Gemini Michelle \\
\hline $12 \mu \mathrm{m} \ldots . .$. & 11.5 & 184 & 26 & $\operatorname{IRAS}$ \\
\hline $24 \mu \mathrm{m} \ldots . .$. & 24.0 & 66.5 & 2.7 & MIPS \\
\hline $60 \mu \mathrm{m} \ldots . .$. & 60.0 & 28 & 10 & ISO \\
\hline $90 \mu \mathrm{m} \ldots . .$. & 90.0 & 26 & 10 & ISO \\
\hline
\end{tabular}

${ }^{\text {a }}$ The standard $B$ and $V$ magnitudes were obtained by converting Tycho $B$ and $V$ magnitudes using Table 2 in Bessell (2000).

$\mathrm{b} B$ and $V$ flux density uncertainties were computed assuming 0.200 for their magnitude uncertainties in order to compensate for some missing opacity species in the model spectrum (see Rhee et al. 2007b). ground with Michelle narrow bands. Furthermore, the reported position of the $M S X$ source is $\sim 12^{\prime \prime}$ west of HD 23514. To reconcile the discrepancy in flux and check the nature of the $\sim 3 \sigma$ positional offset, we downloaded a $2^{\circ} \times 2^{\circ} M S X$ A-band image of HD 23514. The image contains about two dozen $M S X$ sources. When we overplotted $M S X$ catalog positions on the MSX A-band image, only HD 23514 shows a mysterious shift of $\sim 12^{\prime \prime}$ from the obvious source in the image. All other MSX catalog positions fall right on bright sources in the image. Given the fact that no source other than HD 23514 appeared in the $32^{\prime \prime} \times 24^{\prime \prime}$ field of Michelle images, we attribute the mysterious $12^{\prime \prime}$ offset to an erroneous astrometric correction of MSX. Although the nominal wavelength of the MSX A band is $8.28 \mu \mathrm{m}$, its effective wavelength is dependent on the source spectrum (hence the need for color correction). When a true source spectrum is very different from the assumed one $\left(F_{\lambda} \sim \lambda^{-1}\right)$ as in our case, the effective wavelength of the MSX A band can be significantly shifted. To quote an MSX measured flux at its nominal wavelength of $8.28 \mu \mathrm{m}$, a color correction needs to be applied that can account for the apparent discrepancy between our narrowband and $M S X$ catalog fluxes.

In Figure 1, the slope of the NIRSPEC KL spectrum agrees with the dust continuum fit we derive below. The $8-13 \mu \mathrm{m}$ spectrum (Figs. 1 and 2) reveals warm small dust grains near HD 23514 through a prominent emission feature. For young stars and debris disks, the most prominent spectral feature in the $N$ band is silicate emission. However, our $N$-band HD 23514 spectrum peaking at $\sim 9 \mu \mathrm{m}$ is different from almost all other frequently seen silicate features that peak at $9-11 \mu \mathrm{m}$ due to various combinations of olivine, pyroxene, and other minerals 
TABLE 2

Main-Sequence Stars with Debris Systems in the Terrestrial Planetary Zone

\begin{tabular}{|c|c|c|c|c|c|c|}
\hline Object & Spectral Type & $\begin{array}{l}\text { Dust Temperature } \\
\text { (K) }\end{array}$ & $\begin{array}{c}\tau \\
\left(\times 10^{-4}\right)\end{array}$ & $\begin{array}{l}\text { Age } \\
(\mathrm{Myr})\end{array}$ & Cold Dust & References \\
\hline HD $23514^{\mathrm{a}} \ldots \ldots \ldots \ldots \ldots \ldots \ldots \ldots$ & F6 V & 750 & 200 & 100 & Maybe & 1 \\
\hline $\mathrm{BD}+20307^{\mathrm{b}} \ldots \ldots \ldots \ldots \ldots \ldots \ldots \ldots$ & G0 V & 650 & 400 & 400 & No & 2 \\
\hline$\zeta$ Lep & A3 & 190 & 0.65 & 300 & No & 3,4 \\
\hline HD $72905^{\mathrm{c}}$ & G1.5 & ? & 1 & 400 & Yes & 5,6 \\
\hline 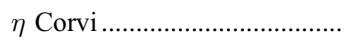 & $\mathrm{F} 2 \mathrm{~V}$ & 180 and 30 & 5 & 600 & Yes & $4,6,7$ \\
\hline 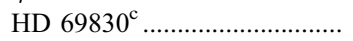 & $\mathrm{K} 0 \mathrm{~V}$ & $?$ & 2 & 2000 & No & 6,8 \\
\hline
\end{tabular}

Nоте.-We define the terrestrial planet zone (TPZ) to be the region where dust particles that radiate like blackbodies will attain a temperature of at least $150 \mathrm{~K}$ (see Rhee et al. 2007a).

${ }^{a}$ Our fit to the SED in Fig. 1 implies a photospheric temperature of $6400 \mathrm{~K}$ and a stellar radius of $1.28 R_{\odot}$ for an assumed distance to HD 23514 of $130 \mathrm{pc}$. However, based on pre-main-sequence evolution models of Baraffe et al. $(1998,2002)$, a $\sim 100$ Myr old $6400 \mathrm{~K}$ star has radius $1.38 R_{\odot}$ and mass $1.35 M_{\odot}$. If HD 23514 is a single star, then the discrepancy between the two radii would be eliminated if the actual distance to HD 23514 is $\sim 140 \mathrm{pc}$. The spectral type of HD 23514 is listed as F5 in Gray et al. (2001), while Cox (2000) gives F7 for a main-sequence star with $T=6400 \mathrm{~K}$. We adopt $\mathrm{F} 6$ in this paper.

${ }^{\mathrm{b}} T=6000 \mathrm{~K}, R=1.25 R_{\odot}$ (Song et al. 2005).

c Following Wyatt et al. (2007), we list HD 69830 and HD 72905 as potentially having dust particles in the TPZ. Beichman et al. (2005) and Lisse et al. (2007) fit a complex model to the mid-IR spectrum of HD 69830 and derive an underlying dust continuum temperature of $400 \mathrm{~K}$. However, given the number of free parameters included and not included (e.g., particle shape), in these models we regard the dust temperature as not well constrained.

REFERENCES.-(1) This paper; (2) Song et al. 2005; (3) Chen \& Jura 2001; (4) Chen et al. 2006; (5) Beichman et al. 2006; (6) Wyatt et al. 2007; (7) Wyatt et al. 2005; (8) Beichman et al. 2005.

(Fig. 2). Possible carriers of this bizarre $9 \mu \mathrm{m}$ emission feature among common minerals in our solar system and Earth's surface are tektosilicates and sulfates. Tektosilicates are a group of light-colored silicate minerals, and this group contains most common minerals (quartz, feldspar, etc.) seen on Earth's surface. About $75 \%$ of Earth's crust is composed of tektosilicates. However, explaining the strong $9 \mu \mathrm{m}$ feature without accompanying prominent olivine and pyroxene signatures is challenging. For example, it is difficult to imagine an extraordinary amount of sulfates at HD 23514 over more commonly appearing minerals unless the chemical composition of HD 23514 is very different from solar. The huge quantity of dust needed to match the HD 23514 spectral energy distribution (SED) can be generated by catastrophic collisions among planetary embryos or even a planet-planet collision (see $\S 3$ ). The latter mimics the postulated Moon-creating collision between the young Earth and a Mars-sized planet (Hartmann \& Davis 1975; Cameron \& Ward 1976). Crustal material ejected from such a collision may naturally explain our HD $23514 \mathrm{~N}$-band spectrum. However, it is hard to explain how crustal material was ejected predominantly over mantle material considering that Earth's crust is a thin layer occupying only $<5 \%$ of volume compared to the mantle. Nonetheless, the unusual $\mathrm{N}$-band spectrum of HD 23514 must bear a clue to the origin of dust, and a wider range mid-IR spectrum is needed for more detailed analysis. At this stage, we likely rule out the case of collisional grinding of many asteroids as the source of dust around HD 23514 since olivine and pyroxene should be the dominant minerals in such environments. We note that few objects out of $111 \mathrm{~T}$ Tauri stars investigated by Furlan et al. (2006), who used Spitzer IRS, show a mid-IR emission feature peaking near $9 \mu \mathrm{m}$ (e.g., IRAS 04187+1927 and CZ Tau), as found in HD 23514.

\section{DISCUSSION}

Excess emission peaking at mid-IR wavelengths indicates that dust must be warm and close to the central star. The temperature, as well as the amount of dust and its distance from the central star, is constrained by creating an SED assuming that dust exists as an optically thin ring. We produced an SED of HD 23514 (Fig. 1) by fitting observed measurements at optical and infrared bands with a stellar photosphere model (Hauschildt et al. 1999) and a single-temperature blackbody of $T=750 \mathrm{~K}$. Large blackbody grains in thermal equilibrium at $750 \mathrm{~K}$ would be located $\sim 0.25$ AU from HD 23514. Even small grains that radiate less efficiently, especially those responsible for the mid-IR emission feature, likely lie within a few AU of the central star. We noted in Rhee et al. (2007a) that stars with warm dust emission, ages between 10 and $30 \mathrm{Myr}$, and spectral types from $\mathrm{G} 0$ to A do not show any evidence in their SEDs of the presence also of cold dust. In such cases, even the small dust particles that carry the strong mid-IR emission feature are likely located close to the stars. Likewise, there is no obvious evidence for cold dust associated with BD +20 307 (Song et al. 2005; A. Weinberger et al. 2008 , in preparation). However, this may not be the case for HD 23514 because Spitzer and ISO points in its SED all lie somewhat above the $750 \mathrm{~K}$ dust continuum as shown in Figure 1, thus suggesting the presence of cooler dust farther from the star. Still, caution is appropriate in interpretation of the Spitzer $25 \mu \mathrm{m}$ flux density measurement as it may be elevated by inclusion of the red wing of a silicate emission feature (e.g., A. Weinberger et al. 2008, in preparation). And the 60 and $90 \mu \mathrm{m} I S O$ points, respectively, lie only 2 and $2.3 \sigma$ above the $750 \mathrm{~K}$ dust continuum line in Figure 1. A Spitzer $70 \mu \mathrm{m}$ flux measurement is highly desirable to clarify the presence of cool dust.

A standard method for characterizing the amount of dust orbiting a star is through the quantity $\tau \equiv L_{\mathrm{IR}} / L_{*}$, where $L_{\mathrm{IR}}$ is the excess luminosity above the photosphere emitted at infrared wavelengths and $L_{*}$ is the bolometric luminosity of the star. We obtained $\tau \sim 2 \times 10^{-2}$ by dividing the infrared excess between 2.3 and $90 \mu \mathrm{m}$ by the stellar bolometric luminosity $\left(2.8 L_{\odot}\right)$; this is $\sim 10^{5}$ times greater than that of the Sun's current zodiacal cloud $\left(\tau \sim 10^{-7}\right)$. HD 23514 thus joins BD +20307 as the two Sun-like main-sequence stars with by far the largest known fractional infrared luminosities (Table 2). The age of BD +20 307 is at least a few hundred million years (Song et al. 2005). As a member of the Pleiades (HII 1132), the age of HD 23514 is 


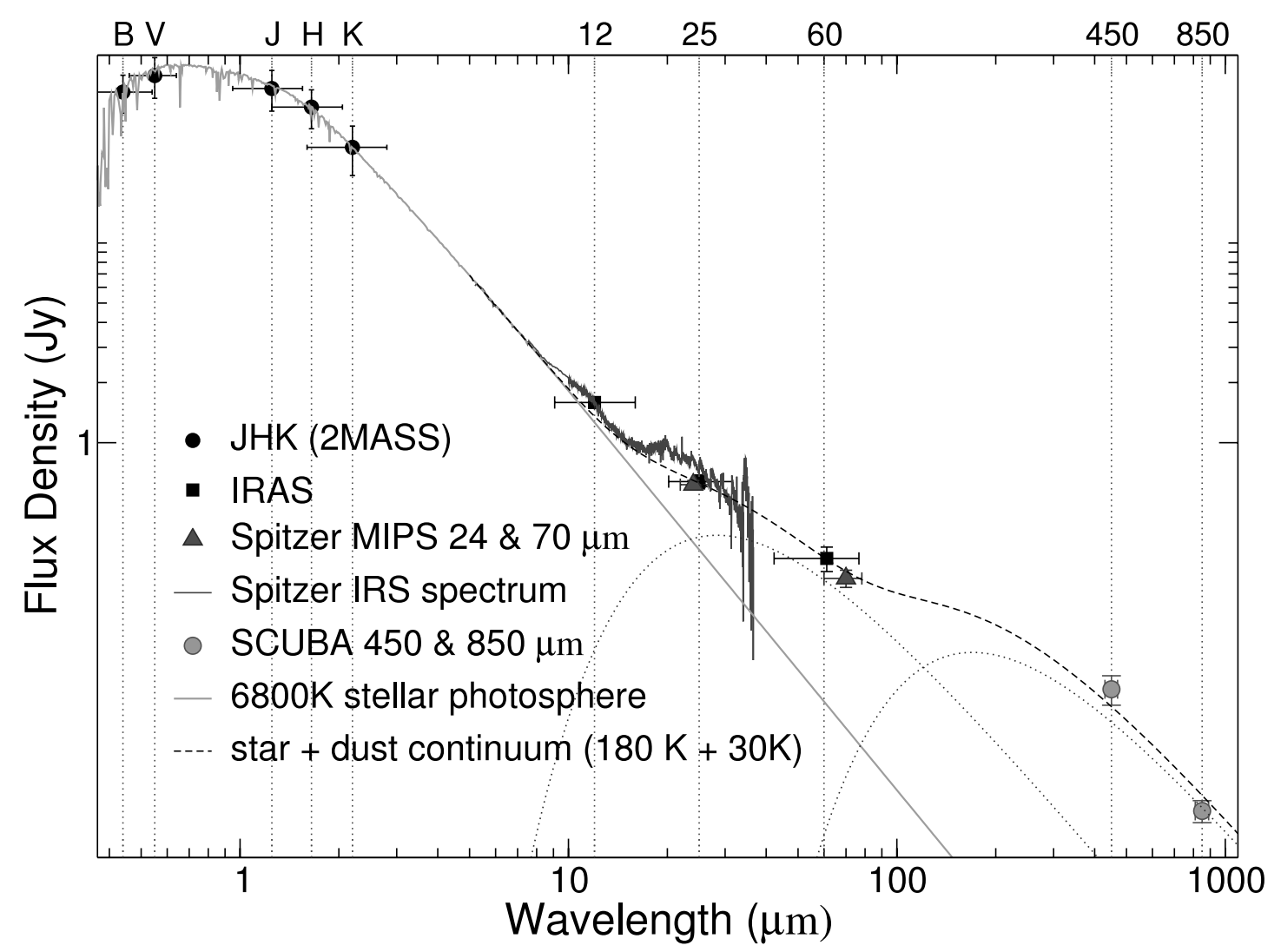

FIG. 3.-SED of $\eta$ Crv. This should replace the SED in Fig. 4 in Wyatt et al. (2005) because (1) we plot IRAS FSC data in preference to the less accurate IRAS PSC data used by Wyatt et al. (2005), and (2) we plot the Spitzer $24 \mu \mathrm{m}$ MIPS measurement. Note that, as a result of these changes, our decomposition of the infrared portion of the SED is very different from that of Wyatt et al. (2005). C. Chen kindly provided the flux-calibrated Spitzer IRS spectrum of $\eta$ Crv. [See the electronic edition of the Journal for a color version of this figure.]

$\sim 100$ Myr. There can be little doubt of cluster membership because in the plane of the sky HD 23514 is located well inside the cluster, ${ }^{4}$ sharing common proper motion, and its radial velocity of $5.9 \pm 0.5 \mathrm{~km} \mathrm{~s}^{-1}$ is in good agreement with the velocity of the Pleiades, $6.0 \pm 1.0 \mathrm{~km} \mathrm{~s}^{-1}$ (Liu et al. 1991).

Currently only a handful of stars with ages $>50$ Myr show warm excess emission ( $T \geqq 150 \mathrm{~K}$ ), indicative of planetesimals in the terrestrial planet zone. Table 2 lists some parameters of these stars. BD +20 307 and HD 23514 stand out among them with very high dust temperature ( $T \geqq 600 \mathrm{~K})$ and $\tau>10^{-2}$. The remaining four warm excess stars have cooler dust and $\tau \sim 10^{-4}$.

The SEDs of HD 23514 and BD +20 307 exhibit excess nearIR emission beginning at wavelengths $\sim 4 \mu \mathrm{m}$. Given the potential importance of stellar wind drag on the dust particles (see below), the absence of really hot dust ( $\gtrsim 1000 \mathrm{~K}$ ) suggests the possible presence of a "sweeper planet" closer to the stars than the dust. Such a situation pertains at HD 69830 where, also, no very hot dust is seen and where Neptune-mass planets interior to the dust disk are known to exist from precision radial velocity measurements (Lovis et al. 2006).

Using a flat disk model, Jura (2003) and Jura et al. (2007) have successfully reproduced the IR emission from flat, geometrically thin dust disks orbiting some white dwarfs. This geometry implies the absence of significant gravitational perturbations by objects with substantial mass located in the vicinity of the dust. In contrast, we find that a flat disk of dust particles generates in-

\footnotetext{
${ }^{4}$ A picture of the Pleiades Cluster with HD 23514 indicated is available from the Gemini Observatory Web site, http://www.gemini.edu/index.php?option= content\&task= view $\& \mathrm{id}=259$.
}

sufficient mid-IR flux to match the SEDs of HD 23514 and $\mathrm{BD}+20307$. Thus, the dust orbiting these stars is puffed up in the vertical direction, perhaps as a result of the gravitational field of the above-mentioned sweeper planet, or the gravity of planetary embryos as discussed below, or both.

Given the ages of HD 23514 and BD +20 307, it is natural to ask whether their huge warm dust burdens were generated by events analogous to those that occurred during the "late heavy bombardment" (LHB) in our solar system. One current model (Gomes et al. 2005) attributes the LHB to a rapid migration of the giant planets that destabilized the orbits of objects in the Kuiper Belt and the main asteroid belt hundreds of millions of years after the formation of the Sun. Wyatt et al. (2007) have proposed a similar model for most of the stars listed in our Table 2, with the difference that they strongly favor an origin of the parent bodies in a region more analogous to the cold Kuiper Belt than the asteroid belt. We note, however, that there is no evidence for cold dust at BD $+20307, \zeta$ Lep, and HD 69830, cold dust that might reasonably be expected at stars with so much warm dust should all parent bodies originate in distant cold regions. The situation is more ambiguous for a Table 2 star like $\eta$ Crv with clear evidence for substantial amounts of cold dust (see Fig. 3). Furthermore, Cuk et al. (2006) argue that LHB was a localized EarthMoon system activity rather than a global, inner solar system event. Thus, because the cause(s) of the LHB remain unsettled, we do not further pursue a relationship between LHB and the high- $\tau$ warm excess phenomenon.

Initially, when there was only one known main-sequence star with very large $\tau\left(>10^{-2}\right)$, the BD +20307 phenomenon might have been regarded as a "miracle," so that a statistical analysis 
of the occurrence rate would have been of questionable value. Now, however, with HD 23514, the frequency of occurrence of such extraordinarily dusty stars can be treated statistically more reliably. BD +20 307 is a field star with estimated age similar to that of the Ursa Majoris moving group (Song et al. 2005), whose age is probably about $400 \mathrm{Myr}$ (Zuckerman et al. 2006). IRAS was sufficiently sensitive to detect main-sequence G-type stars with $\tau>10^{-2}$ out to $\sim 150$ pc. There are $\sim 18,400$ Hipparcos dwarfs with spectral types between F4 and $\mathrm{K} 0$ out to $130 \mathrm{pc}$, the distance to the Pleiades. But most of these are old stars. If the star formation rate was approximately uniform during the past $5 \mathrm{Gyr}$, then there are $\sim 1800$ Hipparcos dwarfs of age $\sim 500 \mathrm{Myr}$ out to $130 \mathrm{pc}$. Then a Hipparcos dwarf with $\tau>10^{-2}$ is found among solar-type field stars about 1 time out of 1500 (after dropping 300 Hipparcos-measured members of nearby stellar clusters).

In the solar vicinity several stellar clusters have solar-type members. We select four rich nearby clusters with ages 70-700 Myr: Hyades, Pleiades, $\alpha$ Persei, and Praesepe. In these stellar clusters, there are $\sim 400$ dwarf stars with spectral types between F4 and K0 (de Bruijne et al. 2001; Stauffer et al. 2007; Lodieu et al. 2005; Adams et al. 2002). Among these, only HD 23514 is identified with $\tau>10^{-2}$. Therefore, the occurrence rate of a dwarf with $\tau>10^{-2}$ in the nearby stellar clusters is, at most, about 1 out of 400. Combining this result with that for BD +20307 indicates that the very high $\tau$, warm dust phenomenon manifests itself in about 1 adolescent star (age a few hundred Myr) in 1000. If all $\mathrm{F} 4-\mathrm{K} 0$ stars display this phenomenon as adolescents, then the lifetime of the phenomenon at a typical solar-like star is a few hundred thousand years.

To interpret our observations, we consider a model of colliding planetary embryos. In a series of papers, Agnor, Asphaug, and colleagues (Agnor et al. 1999; Agnor \& Asphaug 2004; Asphaug et al. 2006) considered the collisions of large bodies in the late stages of the formation of planets in the terrestrial planet zone. Based on their models and those they attribute to earlier researchers (e.g., G. W. Wetherill), we may draw the following conclusions. The process of terrestrial planet formation involves the formation of a minimum of many hundreds of planetary embryos of dimensions $\gtrsim 1000 \mathrm{~km}$. These collide and either they coalesce or, oftentimes, the smaller embryo fragments into smaller objects along with the ejection of "copious debris." While the mass spectrum of the fragments is not well constrained, no large monoliths survive following disruption of large solid bodies. Rather, a typical large fragment size might be $\sim 100 \mathrm{~m}$. Collisions of planetary embryos continue for as long as a few hundred Myr, i.e., to the ages of HD 23514 and BD +20 307; in the following discussion, we assume these to be 100 and $400 \mathrm{Myr}$, respectively. Additional discussion of catastrophic fragmentation of planetary system bodies of moderate size may be found in Fujiwara (1980) and Housen \& Holsapple (1990).

For these assumed ages and an occurrence rate of 1 in 1000 stars, we use a lifetime of 250,000 yr for the HD 23514/BD +20 307 phenomenon at a typical adolescent-age solar-type star. Small particles now in orbit around these two stars will be lost in a much shorter time span and must be replenished many times over. One possible loss mechanism is a collisional cascade that breaks particles down in size until, when their radii become as small as a few tenths of a micron, they become subject to radiation pressure blowout. Other loss mechanisms are PoyntingRobertson (PR) and stellar wind drag. As mentioned above, we assume that the initial mass spectrum is a result of the collision of two planetary embryos, but the spectrum of the collision fragments is not well characterized. Therefore, we assume that col- lisions are sufficiently frequent to establish an approximately equilibrium size distribution:

$$
N(a) d a=N_{o} a^{-3.5} d a
$$

where $N(a)$ is the number of particles per $\mathrm{cm}^{3}$ with radii between $a$ and $a+d a$ (see, e.g., Dohnanyi 1969; Williams \& Wetherill 1994; Chen \& Jura 2001). The smallest particle radius in this distribution may be set by radiation pressure blowout; for the mass $\left(1.35 M_{\odot}\right)$ and luminosity $\left(2.8 L_{\odot}\right)$ we estimate for HD 23514 , this radius is $\sim 0.5 \mu \mathrm{m}$.

With this size distribution most of the mass $(M)$ is carried by the largest particles, while most of the surface area $(\tau)$ is due to small particles with radii not much larger than the submicronsize blowout radius. Specifically,

$$
\begin{gathered}
M \sim \int \frac{4 \pi}{3} a^{3} a^{-3.5} d a \sim a^{1 / 2}, \\
\tau(a) \sim \int N(a) \pi a^{2} d a \sim \int \pi a^{2} a^{-3.5} d a \sim a^{-1 / 2},
\end{gathered}
$$

and

$$
t_{c}=P / \tau \sim a^{1 / 2}
$$

where $t_{c}$ is the collisional lifetime and $P$ is the orbital period. Thus, the smallest particles collide the fastest, for HD 23514 in about 50 yr at 1 AU. Larger objects take longer to collide destructively. They will then be broken down into smaller fragments such that after a collision of two roughly equal mass objects the largest leftover fragment has a radius about $\frac{1}{2}$ that of a collider (S. Kenyon 2007, private communication). As more mass is carried by the larger colliders, with the above $N(a)$, the ratio of mass to collision time is independent of $a$, and there will thus be a supply of material approximately constant with time as the largest objects are eventually whittled down to micron-sized dust. In the Agnor/Asphaug picture outlined above, the largest initial fragments of a collision of planetary embryos might have $a \sim 100 \mathrm{~m}$. Thus, if the lifetime of $a=1 \mu \mathrm{m}$ particles is $\sim 50 \mathrm{yr}$ due either to collisions or to stellar wind drag (see below), then the lifetime of $100 \mathrm{~m}$ fragments will be $\sim 500,000 \mathrm{yr}$. Thus, catastrophic disruption of large planetary embryos (see mass estimate below) can supply material for a time equal to the $250,000 \mathrm{yr}$ event lifetime indicated by our observations.

In addition to collisions, PR drag and stellar wind drag are loss mechanisms for small dust particles. The timescale for PR drag at HD 23514 may be evaluated using, for example, equation (5) in Chen \& Jura (2001). We assume $L_{*}=2.8 L_{\odot}$ (corresponding to a distance of 130 pc to HD 23514), a dust particle orbital semimajor axis of $1 \mathrm{AU}$, and a radius and density of a typical individual grain to be $1 \mu \mathrm{m}$ and $2.5 \mathrm{~g} \mathrm{~cm}^{-3}$, respectively. Then the lifetime against PR drag is $\sim 1000$ yr. For the presentday Sun, PR drag acts about 3 times more rapidly than drag due to the solar wind (Plavchan et al. 2005). However, Wood et al. (2002) estimated that the winds of solar-type stars decline as time to the $2.00 \pm 0.52$ power beginning at ages $\sim 10 \%$ of the current age of the Sun. More recent observation and analysis somewhat cloud quantitative representation of the wind strength as a function of time (Wood et al. 2005; Wood 2006). Based on these references, we assume that, for adolescent-age stars like HD 23514 and BD +20 307, wind drag will dominate PR drag by a factor of 10-30. Then the lifetime of the small particles 
considered above against wind drag will be only $\sim 50 \mathrm{yr}$, i.e., comparable to the collision times. Because PR and wind drag times are proportional to $a$, while the collision time is proportional to $a^{1 / 2}$, the orbital lifetimes of large particles (rocks) are determined by collisions.

To determine how rapidly mass is lost due to either stellar wind drag or collisions, we estimate the minimum dust mass $\left(M_{\min }\right)$ needed to intercept $2 \%$ of the light emitted by HD 23514 . This is

$$
M_{\min }=16 \pi \tau a \rho R^{2} / 3 \text {, }
$$

with $\tau=0.02, a=1 \mu \mathrm{m}, \rho=2.5 \mathrm{~g} \mathrm{~cm}^{-3}$, and $R=1 \mathrm{AU}$. Then $M_{\min } \sim 2 \times 10^{22} \mathrm{~g}$, with a corresponding mass-loss rate, $\dot{M} \sim$ $10^{13} \mathrm{~g} \mathrm{~s}^{-1}$. For BD $+20307, \tau$ is twice as large, so $\dot{M}$ is $\sim 2 \times$ $10^{13} \mathrm{~g} \mathrm{~s}^{-1}$. In $250,000 \mathrm{yr}$, the total mass lost per star will be $\sim 10^{26} \mathrm{~g}$, the mass of Earth's Moon, or, for the above assumed average density, an object with radius $\sim 2000 \mathrm{~km}$. Of course, this mass need not all be produced in one single catastrophic collision, but might rather be a consequence of multiple collisions of smaller planetary embryos spaced over hundreds of millions of years.

\section{CONCLUSIONS}

We show that substantial quantities of warm, small dust particles orbit HD 23514, a solar-type member of the $100 \mathrm{Myr}$ old Pleiades. A similar phenomenon was previously reported (Song et al. 2005) for the somewhat older, solar-type field star BD +20 307. Models for catastrophic collisions of planetary embryos orbiting $\sim 100$ Myr old stars (e.g., Kenyon \& Bromley 2005; Asphaug et al. 2006) will naturally produce such warm dusty disks. Our data are consistent with these model predic- tions, provided that such catastrophic events followed by a subsequent collisional cascade convert of order the mass equivalent of Earth's Moon to tiny dust particles during the early lifetime of many (perhaps most) Sun-like stars. For example, in the case of our solar system, by itself the collision that is postulated to have generated the Moon likely would have sent a comparable mass of debris into interplanetary orbits. Infrared data for stars such as HD 23514 and BD +20 307 are consistent with and may well validate the standard picture of violent formation of terrestriallike planets in the early years of planetary systems.

We thank A. Weinberger and E. E. Becklin for permission to use some Michelle data obtained during a joint Keck time exchange observing run, and $\mathrm{E}$. Rice for assistance in obtaining the NIRSPEC spectrum. We also thank S. Kenyon and C. Lisse each for a helpful discussion and are grateful to C. H. Chen for providing a Spitzer IRS spectrum of $\eta$ Corvi. We appreciate the constructive comments of the referee. Based on observations (GN-2006A-Q-39 and GN-2006B-DD-5) obtained at the Gemini Observatory, which is operated by the Association of Universities for Research in Astronomy, Inc., under a cooperative agreement with the NSF on behalf of the Gemini partnership: the National Science Foundation (United States), the Science and Technology Facilities Council (United Kingdom), the National Research Council (Canada), CONICYT (Chile), the Australian Research Council (Australia), CNPq (Brazil), and SECYT (Argentina). This research was supported in part by NASA grants to UCLA. This research has made use of the VizieR and of data products from the Two Micron All Sky Survey.

\section{REFERENCES}

Adams, J. D., Stauffer, J. R., Skrutskie, M. F., Monet, D. G., Portegies Zwart,

S. F., Janes, K. A., \& Beichman, C. A. 2002, AJ, 124, 1570

Agnor, C., \& Asphaug, E. 2004, ApJ, 613, L157

Agnor, C. B., Canup, R. M., \& Levison, H. F. 1999, Icarus, 142, 219

Asphaug, E., Agnor, C. B., \& Williams, Q. 2006, Nature, 439, 155

Baraffe, I., Chabrier, G., Allard, F., \& Hauschildt, P. H. 1998, A\&A, 337, 403 2002, A\&A, 382, 563

Beichman, C. A., et al. 2005, ApJ, 626, 1061

2006, ApJ, 639, 1166

Bessell, M. S. 2000, PASP, 112, 961

Cameron, A. G. W., \& Ward, W. R. 1976, Lunar and Planetary Institute

Conference Abstracts, 7, 120

Chen, C. H., \& Jura, M. 2001, ApJ, 560, L171

Chen, C. H., et al. 2006, ApJS, 166, 351

Cox, A. N., ed. 2000, Allen's Astrophysical Quantities (4th ed.; New York: AIP)

Cuk, M., Gladman, B. J., \& Gallant, J. 2006, BAAS, 38, 616

de Bruijne, J. H. J., Hoogerwerf, R., \& de Zeeuw, P. T. 2001, A\&A, 367, 111

Dohnanyi, J. W. 1969, J. Geophys. Res., 74, 2531

Fujiwara, A. 1980, Icarus, 41, 356

Furlan, E., et al. 2006, ApJS, 165, 568

Gomes, R., Levison, H. F., Tsiganis, K., \& Morbidelli, A. 2005, Nature, 435, 466

Gray, R. O., Napier, M. G., \& Winkler, L. I. 2001, AJ, 121, 2148

Hartmann, W. K., \& Davis, D. R. 1975, Icarus, 24, 504

Hauschildt, P. H., Allard, F., \& Baron, E. 1999, ApJ, 512, 377

Housen, K. R., \& Holsapple, K. A. 1990, Icarus, 84, 226

Jura, M. 2003, ApJ, 582, 1032

Jura, M., Farihi, J., \& Zuckerman, B. 2007, ApJ, 663, 1285

Kenyon, S. J., \& Bromley, B. C. 2005, AJ, 130, 269

Lisse, C. M., Beichman, C. A., Bryden, G., \& Wyatt, M. C. 2007, ApJ, 658, 584
Liu, T., Janes, K. A., \& Bania, T. M. 1991, ApJ, 377, 141

Lodieu, N., McCaughrean, M. J., Barrado Y Navascués, D., Bouvier, J., \& Stauffer, J. R. 2005, A\&A, 436, 853

Lovis, C., et al. 2006, Nature, 441, 305

McLean, I. S., et al. 1998, Proc. SPIE, 3354, 566

Melis, C., Zuckerman, B., Song, I., Rhee, J., \& Metchev, S. 2008, ApJ, submitted

Meyer, M. R., et al. 2004, ApJS, 154, 422

Plavchan, P., Jura, M., \& Lipscy, S. J. 2005, ApJ, 631, 1161

Rhee, J. H., Song, I., \& Zuckerman, B. 2007a, ApJ, 671, 616

Rhee, J. H., Song, I., Zuckerman, B., \& McElwain, M. 2007b, ApJ, 660, 1556

Song, I., Weinberger, A. J., Becklin, E. E., Zuckerman, B., \& Chen, C. 2002, AJ, 124, 514

Song, I., Zuckerman, B., Weinberger, A. J., \& Becklin, E. E. 2005, Nature, 436, 363

Spangler, C., Sargent, A. I., Silverstone, M. D., Becklin, E. E., \& Zuckerman, B. 2001, ApJ, 555, 932

Stauffer, J. R., et al. 2007, ApJS, 172, 663

Williams, D. R., \& Wetherill, G. W. 1994, Icarus, 107, 117

Wood, B. E. 2006, Space Sci. Rev., 126, 3

Wood, B. E., Müller, H.-R., Zank, G. P., \& Linsky, J. L. 2002, ApJ, 574, 412

Wood, B. E., Müller, H.-R., Zank, G. P., Linsky, J. L., \& Redfield, S. 2005, ApJ, 628, L143

Wyatt, M. C., Greaves, J. S., Dent, W. R. F., \& Coulson, I. M. 2005, ApJ, 620, 492

Wyatt, M. C., Smith, R., Greaves, J. S., Beichman, C. A., Bryden, G., \& Lisse, C. M. 2007, ApJ, 658, 569

Zuckerman, B., Bessell, M. S., Song, I., \& Kim, S. 2006, ApJ, 649, L115

Zuckerman, B., et al. 2007, ApJ, submitted 